Proceedings of the International Symposium on Physics of Materials (ISPMA 14), September 10-15, 2017, Prague

\title{
Modeling of Double Cross-Slip by Means of Geodesic Curvature Driven Flow
}

\author{
M. Kolár, M. Beneš, J. Kratochvíl, P. Pauš \\ ${ }^{a}$ Department of Mathematics, Faculty of Nuclear Sciences and Physical Engineering, \\ Czech Technical University in Prague, Trojanova 13, Prague, Czech Republic \\ ${ }^{b}$ Department of Physics, Faculty of Civil Engineering, Czech Technical University in Prague, \\ Thákurova 7, Prague, Czech Republic
}

\begin{abstract}
In this paper we develop a model for double cross-slip in fcc crystals. The double cross-slip mechanism is demonstrated by a simulation of an overcoming of a particle exerting spherically symmetric repulsive stress field. The cross-slip is treated as a deterministic, stress-controlled process. For the identification of the cross-slip, we use a criterion based on evaluation of stresses exerted on a tip of a screw part of a dislocation resolved in the primary plane and in the cross-slip plane. The motion of a dislocation is described by the geodesic curvature driven flow on surfaces, and treated by means of the parametric approach. The results of numerical simulations are validated by analytical calculations.
\end{abstract}

DOI: 10.12693/APhysPolA.134.667

PACS/topics: 02.70.Bf, 61.72.Lk, 02.30.Jr

\section{Model}

A typical family of crystallographic planes of slip systems of fcc metals, e.g. $\mathrm{Cu}$, is of the $\{111\}$ type. A dislocation motion is planar, however, screw segments can switch from one plane of the $\{111\}$ type to another. This effect is called the cross-slip. It is schematically illustrated in the first three stages of Fig. 1a, where a dislocation curve changes from (111) plane to (1111) plane containing the same Burgers vector.

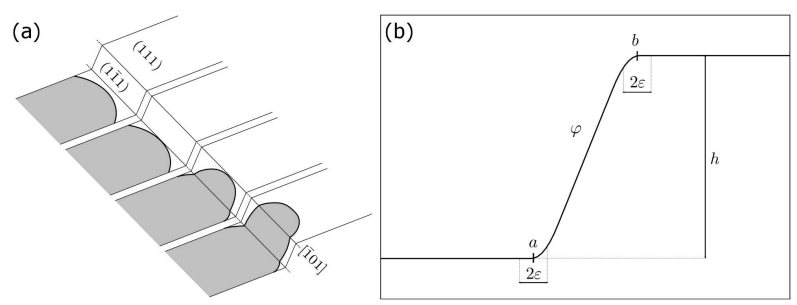

Fig. 1. Double cross-slip illustration. (a) Double crossslip mechanism, (b) cross-slip plane regularization.

Even though the cross-slip is a well known experimentally verified process, it is still considered as an open problem in the field of discrete dislocation dynamics (DDD). So far, in most modeling efforts, the cross-slip is considered as a stochastic, thermally-activated process [1]. In the present paper, we use the approach based on the stress criterion proposed by Kratochvíl and Pauš in $[2,3]$, where the cross-slip is modeled as a deterministic, stress driven process.

corresponding author; e-mail: kolarmir@fjfi.cvut.cz
The criterion presented in [2] compares the shear stresses $\tau_{p}$ and $\tau_{c s}$ exerted on the tip of the screw part of a dislocation resolved in the primary plane and the cross-slip plane, respectively,

1. cross-slip occurs, if $\tau_{c s}>\tau_{p}$,

2. gliding in the primary plane continues, if $\tau_{c s}<\tau_{p}$, where the stresses exerted on the tip are

$$
\tau_{p}=\tau_{\text {obst }}+\tau_{\text {app }}, \quad \tau_{c s}=\tau_{\text {obst }}^{(c s)}+\tau_{\text {app }} / 3 .
$$

Here $\tau_{\text {obst }}$ represents the resolved shear stress exerted by a particle provided the dislocation motion continues in the primary slip plane, $\tau_{a p p}$ is the applied shear stress resolved in the primary slip plane, and $\tau_{\text {obst }}^{(c s)}=\tau_{\text {obst }} \cos 71^{\circ}$ is the shear stress resolved in the cross-slip plane exerted by the particle.

The cross-slip plane is inclined with the angle $\approx 71^{\circ}$ to the primary plane in agreement with the fcc lattice configuration. In the evaluation of the applied stress $\tau_{a p p}$, one has to take into account that in the case of (111) plane, the angle between the loading axis and the normal to the primary plane is $\pi / 4$ and the angle between the primary plane and the cross-slip plane is approximately $71^{\circ}$. The derivation is obtained by means of the Schmidt factor and can be found in the work [2].

In this paper we investigate the cross-slip effects caused by the repulsive stress field exerted by a particle. This serves as an illustrative example demonstrating the expected behavior of the proposed numerical algorithm. Our approach to the cross-slip modeling is based on the mathematical theory of curves moving along a smooth surface of general shape given by the graph of a function $\varphi$. For the technical details on this approach we refer the reader to, e.g. [4]. However, the physical nature of double cross-slip suggests that the given surface is piece- 
wise planar, i.e., non-smooth. To overcome this difficulty, one has to regularize the surface as in Fig. 1b, where the edges between the primary plane and the cross-slip plane were replaced by rounded strips of the width $2 \varepsilon$.

In our understanding of the DDD modeling, dislocations are represented by curves moving by the (mean) curvature (see [5] or [6]) which schematically reads as

normal velocity $=$ curvature + force.

For the dislocation $\mathcal{G}_{t}$ which is allowed to move along several slip planes collectively denoted as $\Sigma$, we propose the motion law generalized to the following form:

$$
B \mathcal{V}_{\mathcal{G}}=-T \mathcal{K}_{\mathcal{G}}+\mathcal{F}
$$

in $\Sigma$, where $B$ is the drag coefficient, $\mathcal{V}_{\mathcal{G}}$ is the normal velocity of the dislocation $\mathcal{G}_{t}$ (here the normal vector $\mathcal{N}$ lies in the tangent space to $\mathcal{G}_{t}$ ), $T$ is the line tension, $\mathcal{K}_{\mathcal{G}}$ is the geodesic curvature of $\mathcal{G}_{t}$. The self-acting force generated by the dislocation is approximated by the product $T \mathcal{K}_{\mathcal{G}}$, where the line tension is calculated in accordance with [7] as $T \approx E^{(e)}\left(1-2 \nu+3 \cos ^{2}(\xi)\right)$, where $E^{(e)}$ is the dislocation edge energy, $\nu$ is the Poisson ratio, and $\xi$ is the angle between the tangent to the dislocation (which lies in the slip plane $\Sigma$ ) and the Burgers vector $\boldsymbol{b}$. Notice that the sign convention for the geodesic curvature is in accordance with, e.g. [8]. The force term $\mathcal{F}$ denotes the sum of all external forces per unit length acting on dislocation $\mathcal{G}_{t}$ in the normal direction $\mathcal{N}$. It is expressed as $\mathcal{F}=b \tau_{\text {res }}$, where $b$ is the magnitude of the Burgers vector and $\tau_{\text {res }}$ is the resolved shear stress acting on $\mathcal{G}_{t}$. For the discussed model of double cross-slip, we consider the following structure of the stress $\tau_{\text {res }}$ :

$$
\tau_{\text {res }}=\tau_{\text {app }}+\tau_{\text {obst }}+\tau_{\text {wall }} \text {. }
$$

Here $\tau_{\text {wall }}$ is the stress exerted by walls of the channel of persistent slip bands (PSB). The PSB channel typically consists of areas with high density of clustered dipolar loops - the channel walls, and areas with low density of dislocations - the channel itself. Walls of the channel are responsible for short-range interactions, and are simulated as the elastic field of infinite edge dipoles. Here one wall of the PSB channel corresponds to one dipole. When a dislocation moves in the PSB channel, the segments near the walls are attracted and then captured in the elastic potential wells created by dipoles. For the scope of our simulation, the PSB channel serves as a convenient boundary for single dislocation curves and in Ref. [9], approximate formulae for the stress $\tau_{\text {wall }}$ exerted by a dipole are presented. The term $\tau_{\text {app }}$ represents the stress applied on the crystal. Our model explores the stress-controlled regime, where $\tau_{\text {app }}$ is considered uniform in the PSB channel. It is known the stress controlled regime slightly overestimates the reality (see [10]). We still believe that this regime is suitable for the geodesic method we use for modelling double cross-slip. The last term $\tau_{\text {obst }}$ is the stress exerted by an obstacle. The obstacle is represented by a particle exerting a spherically symmetric repulsive stress acting on the dislocation. The particle is positioned in the middle of the channel. The criterion for the cross slip is evaluated at the tip of the dislocation. Such a choice of the geometry enables the model validation by analytical calculations. The repulsive stress at a position $\boldsymbol{x}$ exerted by the particle was chosen nonlinear as

$$
\tau_{\text {obst }}=\frac{\tau_{\text {ref }}}{\sqrt{r}} \mathrm{MPa},
$$

where $\tau_{\text {ref }}$ is the reference constant representing the strength of the particle, and $r$ is the distance between the particle and the position $\boldsymbol{x}$. This stress field forces the dislocation to perform the double cross-slip according to the mechanism illustrated in Fig. 1a. Then, the dislocation continues gliding in the parallel primary plane.

\section{Parametric method}

Motion law (1.1) is treated by means of the direct method (also known as the parametric or the Lagrangian method - see [4]). The dislocation curve $\mathcal{G}_{t}$ evolving on a surface given by the graph of a smooth function $\varphi$ is parametrized by means of the vectorial maping $\boldsymbol{X}: I_{u} \times I_{t} \rightarrow \mathbb{R}^{2}$ as the following:

$$
\mathcal{G}_{t}=\left\{(\boldsymbol{X}, \varphi(\boldsymbol{X}))^{T}: \boldsymbol{X} \in \Gamma_{t}\right\},
$$

where $\boldsymbol{X}=\boldsymbol{X}(u, t), I_{u}=[0,1]$ is the fixed interval for spatial parametrization, $I_{t}=\left[0, T_{\max }\right]$ is the time interval, and the planar curve $\Gamma_{t}$ is the vertical projection of $\mathcal{G}_{t}$ to the primary plane, i.e., $\Gamma_{t}=\operatorname{Image}(\boldsymbol{X}(u, t))$. In our approach, we analyze the flow of surface curves $\mathcal{G}_{t}$ driven by motion law (1.1) by means of the flow of projected planar curves $\Gamma_{t}$. In the following text, we summarize the system of governing equations for the mapping $\boldsymbol{X}(u, t)$ of $\Gamma_{t}$ provided $\mathcal{G}_{t}$ glides in its normal direction to the dislocation line in its glide plane by (1.1). For technical details on this approach, we refer the reader to, e.g. [4, 8].

The geometrical quantities of our interest are given by means of the parametrization $\boldsymbol{X}$. The unit tangential vector to $\Gamma_{t}$ is expressed as $\boldsymbol{t}_{\Gamma}=\partial_{u} \boldsymbol{X} /\left|\partial_{u} \boldsymbol{X}\right|$, and the unit normal vector to $\Gamma_{t}$ is given as $\boldsymbol{n}_{\Gamma}=\partial_{u} \boldsymbol{X}^{\perp} /\left|\partial_{u} \boldsymbol{X}^{\perp}\right|$, where $\perp$ is the symbol of perpendicularity. The vector $\boldsymbol{n}_{\Gamma}$ is oriented is such a way that $\operatorname{det}\left(\boldsymbol{n}_{\Gamma}, \boldsymbol{t}_{\Gamma}\right)=1$ holds. Using the Frenet formulae, the curvature $\kappa_{\Gamma}$ of $\Gamma_{t}$ is the inner product

$$
\kappa_{\Gamma}=-\frac{1}{\left|\partial_{u} \boldsymbol{X}\right|} \frac{\partial}{\partial_{u}}\left(\frac{\partial_{u} \boldsymbol{X}}{\left|\partial_{u} \boldsymbol{X}\right|}\right) \cdot \boldsymbol{n}_{\Gamma} .
$$

The normal velocity of $\Gamma_{t}$ is then $v_{\Gamma}=\partial_{t} \boldsymbol{X} \cdot \boldsymbol{n}_{\Gamma}$.

Having a curve $\mathcal{G}_{t}$ on a surface given by the function $\varphi$, we are able to express the unit tangent and normal vectors $\mathcal{T}$ and $\mathcal{N}$ from the tangent space to $\mathcal{G}_{t}$, and the geodesic curvature $\mathcal{K}_{\mathcal{G}}$ of $\mathcal{G}_{t}$ in terms of the quantities $\boldsymbol{n}_{\Gamma}, \boldsymbol{t}_{\Gamma}, \kappa_{\Gamma}$, and $\nabla \varphi($ see $[8])$ :

$$
\begin{gathered}
\mathcal{T}=\frac{\left(\boldsymbol{t}_{\Gamma}, \nabla \varphi \cdot \boldsymbol{t}_{\Gamma}\right)^{\mathrm{T}}}{\sqrt{1+\left(\nabla \varphi \cdot \boldsymbol{t}_{\Gamma}\right)^{2}}}, \\
\mathcal{N}= \\
\frac{\left(\left(1+\left(\nabla \varphi \cdot \boldsymbol{t}_{\Gamma}\right)^{2}\right) \boldsymbol{n}_{\Gamma}-\left(\nabla \varphi \cdot \boldsymbol{t}_{\Gamma}\right)\left(\nabla \varphi \cdot \boldsymbol{n}_{\Gamma}\right) \boldsymbol{t}_{\Gamma}, \nabla \varphi \cdot \boldsymbol{n}_{\Gamma}\right)^{\mathrm{T}}}{\sqrt{\left(1+|\nabla \varphi|^{2}\right)\left(1+\left(\nabla \varphi \cdot \boldsymbol{t}_{\Gamma}\right)^{2}\right)}}
\end{gathered}
$$


and the geodesic curvature $\mathcal{K}_{\mathcal{G}}$ of the curve $\mathcal{G}_{t}$ is given by

$\mathcal{K}_{\mathcal{G}}=\frac{\left(1+|\nabla \varphi|^{2}\right)^{1 / 2} \kappa_{\Gamma}-\frac{\boldsymbol{t}_{\Gamma}^{\mathrm{T}} \nabla^{2} \varphi \boldsymbol{t}_{\Gamma}}{\left(1+|\nabla \varphi|^{2}\right)^{1 / 2}}\left(\nabla \varphi \cdot \boldsymbol{n}_{\Gamma}\right)}{\left(1+\left(\nabla \varphi \cdot \boldsymbol{t}_{\Gamma}\right)^{2}\right)^{3 / 2}}$.

Finally, the normal velocity of $\mathcal{G}$ is given as $\mathcal{V}_{\mathcal{G}}=$ $\left(\partial_{t} \boldsymbol{X}, \varphi(\boldsymbol{X})\right)^{T} \cdot \mathcal{N}$.

We assume that $\Gamma_{t}$ is the vertical projection of the dislocation $\mathcal{G}_{t}$. Then, we seek for a geometric equation for the normal velocity $v_{\Gamma}$. One can easily derive (see [8]) the relation between the normal velocities $\mathcal{V}_{\mathcal{G}}$ and $v_{\Gamma}$ :

$$
\mathcal{V}_{\mathcal{G}}=\sqrt{\frac{1+|\nabla \varphi| 2}{1+\left(\nabla \varphi \cdot \boldsymbol{t}_{\Gamma}\right)^{2}}} v_{\Gamma} .
$$

Then, the dislocation $\mathcal{G}_{t}$ moves according (1.1) provided the parametrization $\boldsymbol{X}$ of its vertical projection satisfies the following system:

$$
\begin{aligned}
& B \partial_{t} \boldsymbol{X}=\alpha T \frac{1}{\left|\partial_{u} \boldsymbol{X}\right|} \frac{\partial}{\partial_{u}}\left(\frac{\partial_{u} \boldsymbol{X}}{\left|\partial_{u} \boldsymbol{X}\right|}\right)+(\beta T+\gamma \mathcal{F}) \frac{\partial_{u} \boldsymbol{X}^{\perp}}{\left|\partial_{u} \boldsymbol{X}\right|}, \\
& \alpha=\frac{1}{1+\left(\nabla \varphi \cdot \boldsymbol{t}_{\Gamma}\right)^{2}}, \\
& \beta=\frac{\left(\boldsymbol{t}_{\Gamma}^{T} \nabla^{2} \varphi \boldsymbol{t}_{\Gamma}\right)\left(\nabla \varphi \cdot \boldsymbol{n}_{\Gamma}\right)}{\left(1+\left(\nabla \varphi \cdot \boldsymbol{t}_{\Gamma}\right)^{2}\right)\left(1+|\nabla \varphi|^{2}\right)}, \\
& \gamma=\sqrt{\frac{1+\left(\nabla \varphi \cdot \boldsymbol{t}_{\Gamma}\right)^{2}}{1+|\nabla \varphi|^{2}}} .
\end{aligned}
$$

For the open dislocation curve, parametric equations are accompanied by the fixed-ends boundary conditions $\boldsymbol{X}_{u=0}=\boldsymbol{X}_{0}, \boldsymbol{X}_{u=1}=\boldsymbol{X}_{1}$ placed in two walls of the PSB channel, and by the initial condition $\boldsymbol{X}_{t=0}=\boldsymbol{X}_{\text {ini }}(u)$, which is a straight line between the fixed endpoints.

Spatial discretization of system (2.5) is obtained by the method of flowing finite volumes, described e.g., in [9]. Then, the semi-implicit time stepping is used and the resulting three-diagonal linear systems are solved by means of the matrix factorization at each time step. To ensure the smoothness of the surface along which the dislocation $\mathcal{G}_{t}$ evolves, the sharp interfaces between primary planes and the cross-slip plane are regularized by a small parameter $\varepsilon$.

\section{Computational results}

In this section we present the results of our computational experiment demonstrating the double cross-slip mechanism. The simulation was performed for fcc crystal copper with idealised particle exerting spherically symmetric repulsive stress. The parameters of the simulation are summarized in Table I.

In the numerical experiment, the particle is positioned at $\left(x_{\text {obst }}, z_{\text {obst }}, y_{\text {obst }}\right)^{\mathrm{T}}=(0,64,-1)^{\mathrm{T}} \mathrm{nm}$ of the $x z y$ coordinate system and the dislocation is surpassed with the repulsive stress

$$
\tau_{\mathrm{obst}}=\frac{-240}{\sqrt{r}} \mathrm{MPa},
$$

where $r$ is the distance from the particle. Notice that the position of the particle slightly below the $x z$ plane causes
Parameters of the simulation.

TABLE I

\begin{tabular}{l|c}
\hline \hline Parameter & Value \\
\hline Burgers vector magnitude & $b=0.256 \mathrm{~nm}$ \\
dislocation edge energy & $E^{(e)}=2.35 \mathrm{nN}$ \\
drag coefficient & $B=1.0 \times 10^{-5} \mathrm{~Pa} \mathrm{~s}$ \\
channel width & $d_{c}=1200 \mathrm{~nm}$ \\
shear modulus & $\mu=42.1 \mathrm{GPa}$ \\
Poisson ratio & $\nu=0.43$
\end{tabular}

a non-symmetric configuration and thus the preferable declination of the cross-slip plane will be upwards. If the dislocation is driven by the applied stress $\tau_{\text {app }}=30 \mathrm{MPa}$, the cross-slip criterion states that the dislocation shifts to the cross-slip plane when $\tau_{c s}>\tau_{p}$, i.e., in the case when the tip of the dislocation and the particle are closer than $r=65.5 \mathrm{~nm}$. When $\tau_{c s} \leq \tau_{p}$, i.e., for greater distance than 65.5 the dislocation returns to the primary plane again. Thus, the expected position of the first cross-slip is at the z-coordinate $z_{a}=-1.49 \mathrm{~nm}$.

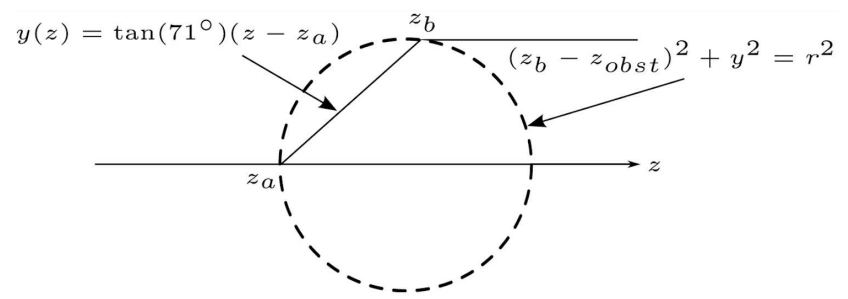

Fig. 2. 1D projection of the dislocation tip and the primary plane and the cross-slip plane. (a)

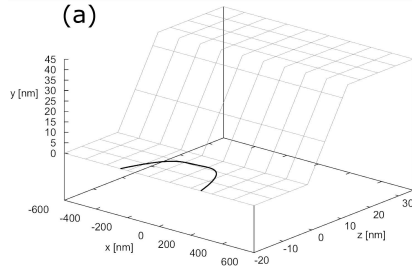

(c)

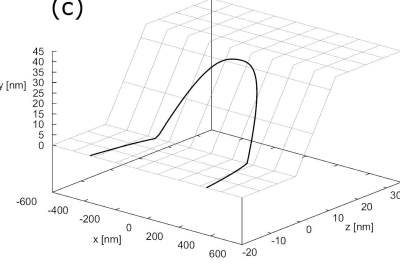

(b)

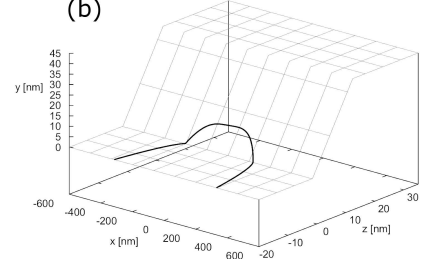

(d)

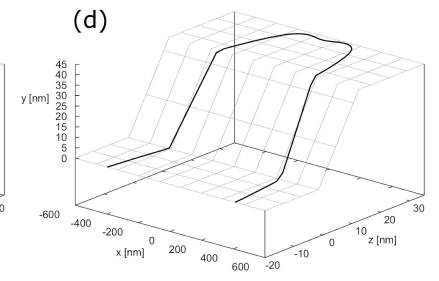

Fig. 3. Detail of the dislocation double cross-slip.

Let us assume for simplicity that the tip of the dislocation moves exactly in the middle of the PSB channel and that the particle exerts spherically symmetric repulsive stress field. Then, the cross-slip can be understood as a 1D problem where the motion of the tip of the dislocation moves along a piece-wise linear graph (see Fig. 2). Here points $z_{a}$ and $z_{b}$ are the coordinates of the first and second cross-slip, and the abscisse 
$y(z)=\tan \left(71^{\circ}\right)\left(z-z_{a}\right)$ represents the cross-slip plane. The circle $\left(z-z_{\text {obst }}\right)^{2}+\left(y-y_{\text {obst }}\right)^{2}=r^{2}$ represents the boundary, where the cross-slip occurs. Thus, the position of the second cross-slip $z_{b}=11.78 \mathrm{~nm}$.

According to our numerical simulation, the first and the second cross-slip occurred in the coordinates $z_{a}=$ $-1.47 \mathrm{~nm}$ and $z_{b}=11.79 \mathrm{~nm}$, which is in good agrement with our analytical calculations. The inaccuracy in the numerical results was caused by space and time discretization. The graphical record of our computational experiment demonstrating the double cross-slip phenomenon are shown in Fig. 3.

\section{Conclusion}

In this article we presented the novel approach to modeling the double cross-slip considered as the deterministic, stress controlled process caused by a particle exerting spherically symmetric repulsive stress field. The method was based on the description of moving surface curves driven by the geodesic curvature, and the deterministic cross-slip criterion comparing stresses resolved in the primary plane and in the cross-slip plane. The method was tested on a simple scenario, where the coordinate of the first and the second cross-slip were calculated analytically as $-1.49 \mathrm{~nm}$, and $11.78 \mathrm{~nm}$, respectively. According to the results of the numerical experiment, the coordinates of both cross-slips are $-1.47 \mathrm{~nm}$ and $11.79 \mathrm{~nm}$, respectively, which indicates a good agreement.

\section{Acknowledgments}

The research has been partly supported by the project No. 14-36566G of the Czech Science Foundation, and the project No. OHK4-001/17 2017-19 of the Student Grant Agency of the Czech Technical University in Prague.

\section{References}

[1] L.P. Kubin, Dislocations, Mesoscale Simulations and Plastic Flow, Oxford University Press, 2013.

[2] P. Pauš, J. Kratochví, M. Beneš, Acta Mater. 61, 7917 (2013).

[3] P. Pauš, J. Kratochvíl, M. Beneš, Philos. Mag. Lett. 94, 45 (2014)

[4] M. Kolář, M. Beneš, D. Ševčovič, Discr. Contin. Dynam. Syst. Series B 22, 28 (2017).

[5] M. Beneš, J. Kratochvíl, J. Křištan, V. Minárik, P. Pauš, Eur. Phys. J. Spec. Topics 177, 177 (2009).

[6] P. Pauš, M. Beneš, M. Kolář, J. Kratochvíl, Modell. Simulat. Mater. Sci. Eng. 24, 035003 (2016).

[7] D. Hull, D. Bacon, Introduction to Dislocations, Butterworth-Heinemann, 2011.

[8] K. Mikula, D. Ševčovič, Comput. Vis. Sci. 6, 211 (2004).

[9] M. Kolář, M. Beneš, D. Ševčovič, J. Kratochvíl, IAENG Int. J. Appl. Math. 45, 198 (2015).

[10] M. Kolář, M. Beneš, J. Kratochvíl, P. Pauš, Acta Phys. Pol. A 128, 506 (2015). 